\title{
Pseudolipoma of the Inferior Vena Cava
}

\author{
Kushaljit Singh Sodhi • Akshay Kumar Saxena • \\ Niranjan Khandelwal • Y. K. Chawla
}

Received: 29 May 2012 / Accepted: 2 December 2012 / Published online: 20 December 2012

(C) Association of Surgeons of India 2012

\begin{abstract}
Pseudolipoma of inferior vena cava refers to a normal variant of adipose collection that is seen in typical pericaval or juxtacaval location; however, it mimics an intracaval mass lesion, especially fat-containing lesions such as lipoma. Awareness of this entity is very important to avoid misdiagnosis and unnecessary diagnostic procedures.
\end{abstract}

Keywords Pseudolipoma $\cdot$ Inferior vena cava $\cdot$ CT scan

\section{Case Report}

A 70-year-old man presented with complaints of abdominal pain. He reported no other complaints and had no history of trauma. Contrast-enhanced computed tomography (CT) of abdomen was performed, which revealed hepatic steatosis with normal pancreas, spleen, kidneys, and gallbladder. Transverse CT scan (Fig. 1) images showed a rounded, well-defined, fat-containing lesion, which initially appeared to lie within the intrahepatic inferior vena cava (IVC). However, sagittal reformation (Fig. 2) of the volume base images was performed, which revealed its true location to be juxtacaval and pericaval rather than intracaval. Based on complete further

\footnotetext{
K. S. Sodhi $(\varangle) \cdot$ A. K. Saxena $\cdot$ N. Khandelwal

Department of Radiodiagnosis, Postgraduate Institute

of Medical Education \& Research (PGIMER),

Chandigarh 160 012, India

e-mail: sodhiks@gmail.com

Y. K. Chawla

Department of Hepatology, Post Graduate Institute of Medical Education \& Research, (PGIMER),

Chandigarh -160 012, India
}

workup, a final diagnosis of peptic ulcer disease was made in this patient.

Pericaval fat or adipose collection mimicking an intracaval mass has been described to occur in $0.5 \%$ of adults undergoing abdominal CT [1]. When present, this localized collection of fat is characteristically seen medially or posteriorly in the lumen of IVC at or superior to the confluence of the hepatic veins and IVC $[2,3]$. It has been suggested that variable appearances of an extracaval fat collection at serial $\mathrm{CT}$ result from differences in respiratory depth or pressure [1].

Ultrasound and reformatted CT images are helpful in identifying its extracaval origin and avoiding it being misdiagnosed as a lipomatous intracaval lesion. Han et al. [2] using helical CT-reformatted images have proposed that this appearance results from the association of juxtacaval fat with angulation and narrowing of the medial aspect of the immediately subdiaphragmatic IVC. This finding at CT scan has been considered benign,

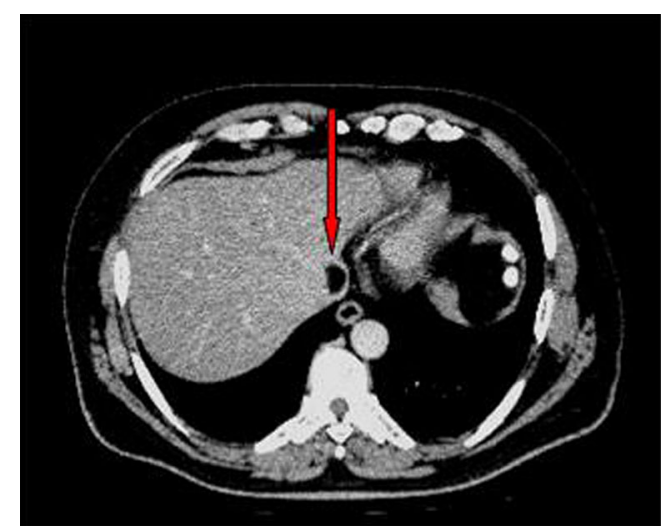

Fig. 1 Transverse CT image reveals as an intracaval fat-containing pseudo-lesion (arrow) 


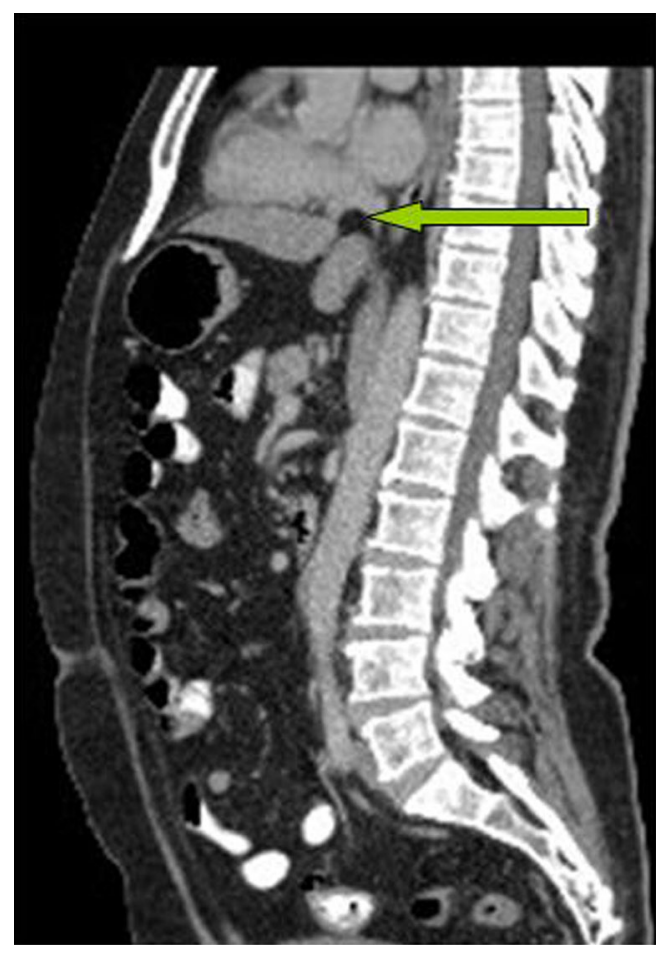

Fig. 2 Sagittal reformatted image shows the fatty lesion (arrow) is juxta- and pericaval in location rare, and incidental process and related to chronic liver disease, anatomical variations, and even obesity [3]. Knowledge and awareness of pericaval and juxtacaval fat collection is important to avoid misdiagnosis and unnecessary diagnostic procedures. CT scan with multiplanar reformation is an ideal tool that often helps in arriving at the correct diagnosis and proving that it is not an intracaval lesion [4].

\section{References}

1. Miyake H, Suzuki K, Ueda S et al (1992) Localized fat collection adjacent to the intrahepatic portion of the inferior vena cava: a normal variant on CT. AJR 158:423-425

2. Han BK, Im JG, Jung JW et al (1997) Pericaval fat collection that mimics thrombosis of the inferior vena cava: demonstration with use of multi-directional reformation CT. Radiology 203:105-108

3. Gibo M, Murata S, Kuroki S (2001) Pericaval fat collection mimicking an intracaval lesion on CT in patients with chronic liver disease. Abdom Imaging 26:492-495

4. Sodhi KS, Ojili V, Sakhuja V, Khandelwal N (2008) Hepatic and inferior vena caval thrombosis: vascular complication of amoebic liver abscess. J Emerg Med 34:155-157 